BESANÇON

Autour des formes trace des algèbres cycliques 


\section{Autour des formes trace des algèbres cycliques}

\section{Grégory Berhuy}

U.M.R.6623 du C.N.R.S., Labo de Maths, Bureau 401M, 16 route de Gray,

F-25030 Besançon, France

(e-mail : berhuy@math.univ-fcomte.fr)

Introduction : Soit $k$ un corps de caractéristique différente de 2. Dans cet article, on calcule les formes trace des algèbres cycliques $(a, L / k, \sigma)$ lorsque $k$ vérifie $I^{3}(k)=0$, ou lorsque $k$ est quelconque et $-1 \in L^{* 2}$. Dans une première partie, on redémontre un théorème de Watanabe (cf.[W]) qui établit le lien entre les formes trace des produits croisés et certaines formes trace hermitiennes amplifiées. On utilise ensuite les résultats connus sur les formes trace des extensions cycliques pour obtenir celles des algèbres cycliques en utilisant le résultat de Watanabe.

\section{Définitions et rappels.}

Soit $k$ un corps de caractéristique différente de 2 , et soit $L / k$ une extension finie séparable. Soit $\lambda \in L^{*}$. On peut associer à $L$ les formes quadratiques suivantes, appelées respectivement forme trace et forme trace amplifiée :

$$
\begin{gathered}
\operatorname{Tr}_{E / k}(\langle 1\rangle): x \mapsto \operatorname{Tr}_{E / k}\left(x^{2}\right) \\
\operatorname{Tr}_{E / k}(\langle\lambda\rangle): x \mapsto \operatorname{Tr}_{E / k}\left(\lambda x^{2}\right)
\end{gathered}
$$

Si $L$ possède une involution $k$-linéaire non triviale $\sigma$ (ce qui implique $[L: k]$ pair), et si $\lambda=\lambda^{\sigma}$, on peut aussi définir une forme trace hermitienne et une forme trace hermitienne amplifiée :

$$
\begin{gathered}
\operatorname{Tr}_{E / k}\left(\langle 1\rangle_{\sigma}\right): E \rightarrow k, x \mapsto \operatorname{Tr}_{E / k}\left(x x^{\sigma}\right) \\
\operatorname{Tr}_{E / k}\left(\langle\lambda\rangle_{\sigma}\right): E \rightarrow k, x \mapsto \operatorname{Tr}_{E / k}\left(\lambda x x^{\sigma}\right)
\end{gathered}
$$

Si $A$ est une algèbre centrale simple sur $k$, on note $\operatorname{Trd}_{A}$ la trace réduite de $A$ (dont on rappelera la définition exacte dans la première partie), et on appelle forme trace de $A$ la forme quadratique $\mathcal{T}_{A}: x \in A \mapsto \operatorname{Trd}_{A}\left(x^{2}\right)$. On rappelle que la dimension d'une algèbre centrale simple est un carré, et on appelle degré de $A$ l'entier $\operatorname{deg}_{A}=\sqrt{\operatorname{dim}_{A}}$. Rappelons enfin la définition 
d'une algèbre cyclique. Soit $L / k$ une extension cyclique de degré $n, \sigma$ un générateur de $\operatorname{Gal}(L / k)$ et $a \in k^{*}$. L'anneau $(a, L / k, \sigma)=\bigoplus_{i=0}^{n-1} L e^{i}$ de multiplication $e^{n}=a$ et $\epsilon \lambda=\sigma(\lambda) e, \lambda \in L$ est appelé une algèbre cyclique. On vérifie facilement que c'est une algèbre centrale simple, contenant $L$ comme sous-corps commutatif maximal.

Pour tout ce qui concerne les algèbres centrales simples et les algèbres à division, on pourra consulter [J], [D], [Sc1] ou [KMRT], par exemple.

\section{Remarques préliminaires.}

On rappelle que si $L / k$ est une extension galoisienne de degré $n$ impair, alors $\operatorname{Tr}_{L / k}(\langle 1\rangle) \simeq n\langle 1\rangle$ (cf.[CP] par exemple). Supposons maintenant que $L / k$ est une extension cyclique de degré pair. Posons $[L: k]=n=2^{r} s$, avec $r \geq 1$ et $s \geq 1$ impair. La correspondance de Galois nous assure l'existence de deux sous-extensions de $L, L_{1}$ et $L_{2}$, cycliques, de degrés respectifs $2^{r}$ et $s$. Les degrés de ces deux extensions étant premiers entre eux, $L_{1}$ et $L_{2}$ sont linéairement disjointes. On a alors $L \simeq L_{1} \otimes L_{2}$ et $\operatorname{Tr}_{L / k}(\langle 1\rangle) \simeq \operatorname{Tr}_{L_{1} / k}(\langle 1\rangle) \otimes \operatorname{Tr}_{L_{2} / k}(\langle 1\rangle)$, et on a donc

$$
\operatorname{Tr}_{L / k}(\langle 1\rangle) \simeq s \operatorname{Tr}_{L_{1} / k}(\langle 1\rangle)
$$

Enfin, soit $\tau$ l'unique élément d'ordre 2 du groupe de Galois de $L / k$ et soit $K$ le sous-corps de $L$ fixé par $\tau$. On a $L=K(\sqrt{b})$, avec $b \in K^{*}-K^{* 2}$. Alors on a :

$$
\begin{gathered}
\operatorname{Tr}_{L / k}(\langle 1\rangle) \simeq<2>\otimes \operatorname{Tr}_{K / k}(\langle 1\rangle) \perp<2>\otimes \operatorname{Tr}_{K / k}(\langle b\rangle) \\
\operatorname{Tr}_{L / k}\left(\langle 1\rangle_{\tau}\right) \simeq<2>\otimes \operatorname{Tr}_{K / k}(\langle 1\rangle) \perp-<2>\otimes \operatorname{Tr}_{K / k}(\langle b\rangle)
\end{gathered}
$$

\section{Formes trace des produits croisés.}

Rappelons tout d'abord la définition d'un produit croisé.

Théorème-Définition 1 : Soit $L / k$ une extension finie galoisienne de degré $n$, de groupe de Galois $G$, et soit $x$ un 2-cocycle normalisé de $G$ à valeurs dans $L^{*}$. On définit sur le $k$-espace vectoriel de dimension $n^{2}$ $(x, L / k)=\bigoplus_{\sigma \in G} L e_{\sigma}$ une multiplication par les formules suivantes :

$e_{\sigma} \lambda=\sigma(\lambda) e_{\sigma}$, pour $\lambda \in L, e_{\sigma} e_{\tau}=x(\sigma, \tau) e_{\sigma \tau}$ (la définition étant étendue à tout élément de $(x, L / k)$ par distributivité). Alors les éléments $\epsilon_{\sigma}$ sont 
inversibles, $e_{\mathrm{Id}}=1$ et $(x, L / k)$ est une algèbre centrale simple déployée par $L$, appelée produit croisé.

Pour une démonstration, on pourra consulter [D], section 12 . On a alors le théorème suivant :

Théorème 2 : (cf.[D], §12) Soit $L$ une extension galoisienne finie de groupe de Galois $G$, et soit une $k$-algèbre centrale simple $A$ contenant $L$ comme sous-corps commutatif maximal. Alors il existe un 2-cocycle normalisé $x$ de $G$ à valeurs dans $L^{*}$, tel que $A=(x, L / k)$.

Définition 1 : Soit $A$ une algèbre centrale simple de degré $n$ sur $k$, et $L$ un corps de déploiement de $A$. On a donc un isomorphisme d'algèbres $\varphi: A \otimes L \stackrel{\sim}{\rightarrow} M_{n}(L)$. La trace réduite d'un élément $a \in A$ est par définition $\operatorname{Trd}_{A}(a)=\operatorname{Tr}(\varphi(a \otimes 1))$, où $\operatorname{Tr}$ désigne la trace usuelle. On montre que cette définition ne dépend pas du choix de $L$ et de $\varphi$ (pour plus de détails, cf.[D] ou [Sc1]). On sait en particulier que, si $L$ est un sous-corps commutatif maximal de $A$, alors l'homomorphisme $A \otimes L \rightarrow M_{n}(L), a \otimes \lambda \mapsto(z \mapsto a z \lambda)$ est un isomorphisme. On a donc $\operatorname{Trd}_{A}(a)=\operatorname{Tr}\left(l_{a}\right)$, où $l_{a}$ désigne la multiplication à gauche par $a$ dans $A$ (vu comme $L$-espace vectoriel de dimension $n$ ).

On suppose maintenant que $A=(x, L / k)$. Soit $\left(e_{\sigma_{i}}\right)$ une $L$-base de ce produit croisé (où les $\sigma_{i}$ sont les éléments de $G$, avec $\sigma_{1}=\mathrm{Id}$ ). On a alors le résultat suivant :

Lemme 1 : Soit $a=\sum \lambda_{i} e_{\sigma_{i}}$. On a alors $\operatorname{Trd}_{A}(a)=\operatorname{Tr}_{L / k}\left(\lambda_{1}\right)$.

Preuve : Il est facile de voir qu'un élément de $A \otimes L$ est une somme d'éléments du type $e_{\sigma_{i}} \otimes \lambda=\left(e_{\sigma_{i}} \otimes 1\right) \lambda=e_{\sigma_{i}} \lambda$. Autrement dit, $A \otimes L$ est le $L$-espace vectoriel à droite engendré par les $e_{\sigma_{i}}$. Pour calculer la trace de la multiplication par $a$, il faut donc écrire les éléments de $A \otimes L$ sous la forme de combinaisons linéaires à droite. On a.

$a e_{\sigma_{j}}=\sum_{i} \lambda_{i} x\left(\sigma_{i}, \sigma_{j}\right) e_{\sigma_{i} \sigma_{j}}=\sum_{i} e_{\sigma_{i} \sigma_{j}}\left(\sigma_{i} \sigma_{j}\right)^{-1}\left(\lambda_{i} x\left(\sigma_{i}, \sigma_{j}\right)\right)=e_{\sigma_{j}} \sigma_{j}^{-1}\left(\lambda_{1}\right)+\cdots$ $\left(\operatorname{car} x\left(\sigma_{j}, \mathrm{Id}\right)=1\right)$. La trace de la multiplication par a est alors $\sum_{j} \sigma_{j}^{-1}\left(\lambda_{1}\right)$, c'est-à-dire $\operatorname{Tr}_{L / K}\left(\lambda_{1}\right)$.

On montre maintenant la proposition suivante, due à Watanabe (cf.[W], proposition 6), de manière légèrement différente, et qui nous sera utile dans la suite pour calculer la forme trace des algèbres cycliques: 
Proposition 1 : Soit $A=(x, L / k)$ un produit croisé, et soit $t$ le nombre d'éléments d'ordre 2 de $G a l(L / k)$.

Alors $\mathcal{T}_{A} \simeq \operatorname{Tr}_{L / k}(\langle 1\rangle) \perp \underset{\tau, o(\tau)=2}{\perp} \operatorname{Tr}_{L / k}\left(\langle x(\tau, \tau)\rangle_{\tau}\right) \perp \frac{n(n-1-t)}{2} \mathbb{H}$.

Preuve : Soient $T=\{\tau \in \operatorname{Gal}(L / k), o(\tau)=2\}$ $H=\{\sigma \in \operatorname{Gal}(L / k), \sigma \neq \mathrm{Id}, o(\sigma) \neq 2\}$, et soient $F=\left\langle e_{\tau}, \tau \in T\right\rangle$ et $G=\left\langle e_{\sigma}, \sigma \in H\right\rangle$. On montre aisément grâce au lemme précédent que $L, F$ et $G$ sont orthogonaux deux à deux. $H$ étant constitué d'éléments d'ordres différents de 1 et 2 , il se partitionne de la manière suivante : $H=X \cup X^{-1}$, où $X^{-1}$ est l'ensemble des inverses des éléments de $X$. On voit alors facilement, toujours grâce au lemme 1 que l'espace engendré par les $e_{\sigma}, \sigma \in X$ est un sous-espace totalement isotrope maximal de $H$. La restriction de $\mathcal{T}_{A}$ à $H$ est donc hyperbolique. Il est clair que la restriction de $\mathcal{T}_{A}$ à $L$ est la forme trace de $L / k$. De plus, le lemme 1 entraîne que les sous-espaces $L e_{\tau}$, où $\tau \in T$ sont orthogonaux deux à deux. Enfin, le fait que $\tau \in T$ soit d'ordre 2 implique l'égalité $\operatorname{Trd}_{A}\left(\lambda e_{\tau} \mu e_{\tau}\right)=\operatorname{Tr}_{L / k}\left(x(\tau, \tau) \lambda \mu^{\tau}\right)$, d'où le résultat annoncé.

Corollaire 1 : (cf.[W]) Soit $A=(a, L / k, \sigma)$ une algèbre cyclique sur $k$. Alors $\mathcal{T}_{A} \simeq \operatorname{Tr}_{L / k}(\langle 1\rangle) \perp<a>\otimes \operatorname{Tr}_{L / k}\left(\langle 1\rangle_{\tau}\right) \perp \frac{n(n-2)}{2} \mathbb{H}$, où $\tau=\sigma^{\frac{n}{2}}$

Preuve : Pour pouvoir utiliser la proposition 1, on va maintenant mettre $A$ sous la forme d'un produit croisé, ce qui est possible d'après le théorème 2. Pour $0 \leq i, j \leq n-1$, posons $x_{a, \sigma}\left(\sigma^{i}, \sigma^{j}\right)=a$ si $i+j \geq n$ et 1 sinon. Alors on peut vérifier que $x_{a, \sigma}$ est un 2-cocycle, et que $(a, L / k, \sigma)$ et $\left(x_{a, \sigma}, L / k\right)$ sont équivalentes dans $B r(k)$ (cf.[D], $\S 12$, Exercice 1 par exemple). Comme elles ont même degré, elles sont isomorphes. L'unique élément d'ordre 2 de $\operatorname{Gal}(L / k)$ étant $\tau=\sigma^{\frac{n}{2}}$, on a $x_{a, \sigma}(\tau, \tau)=a$, et donc

$\mathcal{T}_{A} \simeq \operatorname{Tr}_{L / k}(\langle 1\rangle) \perp<a>\otimes \operatorname{Tr}_{L / k}\left(\langle 1\rangle_{\tau}\right) \perp \frac{n(n-2)}{2} \mathbb{H}$, d'après la proposition 1 . 


\section{Quelques cas particuliers.}

Dans ce paragraphe, on donne des résultats qui sont valables sur n'importe quel corps.

Lemme 2 : Soit $k$ un corps de caractéristique différente de 2 . Soit $L / k$ une extension cyclique de degré $n=2^{r} s$, avec $r \geq 0$ et $s \geq 1$ impair. Soit $D$ un représentant de la classe de $\operatorname{disc}(L / k)$ modulo $k^{* 2}$. Alors

$\operatorname{Tr}_{L / k}(\langle 1\rangle) \simeq\left\{\begin{array}{l}n\langle 1\rangle \text { si } r=0 \\ s\langle 2,2 D\rangle \text { si } r=1 \\ s\langle 1, D, q, q\rangle \text { pour un certain } q \in k^{*} \text { si } r=2 \\ s\langle 1,1,1, D\rangle \perp s\langle t\rangle \otimes\langle 1,2, D, 2 D\rangle \\ \text { pour un certain } t \in k^{*} \text { si } r=3\end{array}\right.$

Preuve : Le cas $r=0$ est déjà connu (cf.[CP]). Pour les autres cas, on peut supposer $s=1$ d'après (1). Le cas $r=1$ est alors un simple calcul et les deux autres cas ont été traités dans [DEK], proposition 8 et proposition 10 .

Corollaire 2 : Soit $k$ un corps de caractéristique différente de 2. Soit $L / k$ une extension cyclique de degré $n=2^{r} s$, avec $r \geq 3$ et $s \geq 1$ impair, soit $\tau$ l'unique élément d'ordre 2 de son groupe de Galois, et soit $D$ un représentant de la classe de $\operatorname{disc}(L / k)$ modulo $k^{* 2}$. Alors

$\operatorname{Tr}_{L / k}\left(\langle 1\rangle_{\tau}\right) \simeq\left\{\begin{array}{l}s\langle 2,-2 D\rangle \text { si } r=1 \\ s\langle 1, D,-q,-q\rangle \text { si } r=2 \\ s\langle 1,1,1, D\rangle \perp s\langle-t\rangle \otimes\langle 1,2, D, 2 D\rangle \text { si } r=3\end{array}\right.$

Preuve : Le cas $r=1$ est un calcul direct. Supposons $r=2$. On garde les notations des préliminaires. D'après une remarque précédente, $K$ et $L$ ont même discriminant. Comme $K / k$ est cyclique de degré $2 s$, on a $\operatorname{Tr}_{K / k}(\langle 1\rangle) \simeq s\langle 2,2 D\rangle$. Posons $\Phi=\langle 2\rangle \otimes \operatorname{Tr}_{K / k}(\langle b\rangle)$. En utilisant (2) et le théorème de simplification de Witt, on obtient $\Phi \simeq s\langle q, q\rangle$. On utilise alors (3). Si $r=3, K / k$ étant une extension cyclique de degré 4 contenue dans $L$, on a par [DEK], proposition 9 et par (1), l'isomorphisme $\operatorname{Tr}_{K / k}(\langle 1\rangle) \simeq s\langle 1,1,2,2 D\rangle$, et donc

$\langle 2\rangle \otimes \operatorname{Tr}_{K / k}(\langle 1\rangle) \simeq s\langle 1,1,1, D\rangle$, en tenant compte du fait que $\langle 2,2\rangle \simeq\langle 1,1\rangle$.

On achève alors la preuve de la même manière. 
Corollaire 3 : Soit $k$ un corps de caractéristique différente de 2. Soit $A=(a, L / k, \sigma)$ une algèbre cyclique de degré $n=2^{r} s$, avec $r \geq 0$ et $s \geq 1$ impair, et soit $D$ un représentant de la classe de $\operatorname{disc}(L / k) \bmod k^{* 2}$. Alors

$$
\mathcal{T}_{A} \simeq\left\{\begin{array}{l}
n\langle 1\rangle \perp \frac{n(n-1)}{2} \mathbb{H} \text { si } r=0 \\
\frac{n}{2}\langle 2,2 a, 2 D,-2 a D\rangle \perp \frac{n(n-2)}{2} \mathbb{H} \text { si } r=1 \\
s\langle 1, a, D, a D\rangle \perp 2 s\langle q,-a q\rangle \perp \frac{n(n-2)}{2} \mathbb{H} \text { si } r=2 \\
s\langle 1, a\rangle \otimes\langle 1,1,1, D\rangle \perp s\langle t,-a t\rangle \otimes\langle 1,2, D, 2 D\rangle \\
\perp 8 s(4 s-1) \mathbb{H} \text { si } r=3
\end{array}\right.
$$

Preuve : Le cas impair est connu (cf.[Se2], Annexe, par exemple), et les autres cas sont une application directe du corollaire 1 et du corollaire 2 .

\section{Forme trace des extensions et des algèbres cycliques sur les corps $k$ tels que $I^{3}(k)=0$.}

Théorème 3 : Soit $k$ un corps de caractéristique différente de 2 tel que $I^{3}(k)=0$, et soit $L / k$ une extension cyclique de degré $n=2^{r} s$, avec $r \geq 3$ et $s \geq 1$ impair. Soit $D$ un représentant de la classe de $\operatorname{disc}(L / k)$ modulo $k^{* 2}$. Alors $\operatorname{Tr}_{L / k}(\langle 1\rangle) \simeq s\langle 2,2 D\rangle \perp\left(2^{r} s-2 s\right)\langle 1\rangle$.

Preuve : Le fait que $D \equiv \operatorname{disc}(L / k) \bmod k^{* 2}$ provient de l'égalité $\operatorname{det}\left(\operatorname{Tr}_{L / k}(\langle 1\rangle)\right)=\operatorname{disc}(L / k)$ dans $k^{*} / k^{* 2}$. On peut se limiter au cas où $s=1$, d'après les préliminaires.

On sait, d'après [DEK], corollaire 3 , que $w_{2}\left(\operatorname{Tr}_{L / k}(\langle 1\rangle)\right)=(2, D)$. Il est bien connu qu'un corps vérifiant $I^{3}(k)=0$ est non ordonné et que toute forme quadratique sur $k$ est entièrement déterminée par sa dimension, son discriminant et son invariant de Hasse (cf. [EL]). On vérifie alors que la forme $\langle 2,2 D\rangle \perp\left(2^{r}-2\right)\langle 1\rangle$ possède les invariants désirés, ce qui achève la preuve.

Corollaire 4 : Soit $k$ un corps de caractéristique différente de 2 vérifiant $I^{3}(k)=0$. Soit $L / k$ une extension cyclique de degré $n=2^{r} s$, avec $r \geq 3$ et $s \geq 1$ impair, soit $\tau$ l'unique élément d'ordre 2 de son groupe de Galois, et soit $D$ un représentant de la classe de $\operatorname{disc}(L / k)$ modulo $k^{* 2}$. Alors $\operatorname{Tr}_{L / k}\left(\langle 1\rangle_{+}\right) \simeq s\langle-2,-2 D\rangle \perp 2 s\langle 1\rangle \perp\left(2^{r-1} s-2 s\right) \mathbb{H}$. 
Preuve : Gardons les notations des remarques préliminaires. L'extension $K / k$ est une extension cyclique de degré $2^{r-1} s$ et contenue dans $L$. D'après une remarque faite plus haut, $K$ et $L$ ont même discriminant. En utilisant alors [DEK], corollaire 4, et la formule (1), on a

$\operatorname{Tr}_{K / k}(\langle 1\rangle) \simeq s\langle 2,2 D\rangle \perp\left(2^{r-1} s-2 s\right)\langle 1\rangle$.

Posons $\Phi=\langle 2\rangle \otimes \operatorname{Tr}_{K / k}(\langle b\rangle)$. On a donc

$s\langle 2,2 D\rangle \perp\left(2^{r} s-2 s\right)\langle 1\rangle \simeq s\langle 1, D\rangle \perp\left(2^{r-1} s-2 s\right)\langle 2\rangle \perp \Phi$ d'après (2). En remarquant que $\langle 2,2\rangle \simeq\langle 1,1\rangle$, on obtient

$\Phi \sim s\langle 2,2 D,-1,-D\rangle+2^{r-1} s\langle 1\rangle$ dans $W(k)$. Puisque $D$ est une somme de deux carrés, on a $\langle D, D\rangle \simeq\langle 1,1\rangle$ et donc

$\Phi \sim\left(2^{r-1} s-4 s\right)\langle 1\rangle+s\langle 2,2 D,-1,-D\rangle+s\langle 1,1\rangle+s\langle D, D\rangle$

$\sim s\langle 1,2, D, 2 D\rangle+\left(2^{r-1} s-4 s\right)\langle 1\rangle$, et comme $\Phi$ est de dimension $2^{r-1} s$, on a $\Phi \simeq s\langle 1,2, D, 2 D\rangle \perp\left(2^{r-1} s-4 s\right)\langle 1\rangle$. On obtient alors le résultat en utilisant (3).

On a alors :

Corollaire 5: Soit $k$ un corps de caractéristique différente de 2 vérifiant $I^{3}(k)=0$. Soit $A=(a, L / k, \sigma)$ une algèbre cyclique de degré $n=2^{r} s$, avec $r \geq 3$ et $s \geq 1$ impair, et soit $D$ un représentant de la classe de $\operatorname{disc}(L / k) \bmod k^{* 2}$.

Alors $\mathcal{T}_{A} \simeq s\langle 2,2 a, 2 D,-2 a D\rangle \perp\left(2^{r} s-2 s\right)\langle 1\rangle \perp \frac{n(n-1)}{2} \mathbb{H}$.

Preuve : En utilisant le corollaire 1 et le corollaire 4, on obtient

$\mathcal{T}_{A} \simeq s\langle 2,2 D\rangle \perp\left(2^{r} s-2 s\right)\langle 1\rangle \perp s\langle-2 a,-2 a D\rangle \perp 2 s\langle a\rangle$

$\perp\left(\frac{n(n-1)}{2}-2 s\right) \mathbb{H}$. En remarquant que $\langle a, a\rangle \simeq\langle 2 a, 2 a\rangle$, on obtient le résultat.

Ce résultat donne en particulier les formes trace des algèbres centrales simples sur les corps locaux et les corps globaux non ordonnés, car toute algèbre centrale simple sur de tels corps est cyclique. 


\section{Formes trace des algèbres cycliques lorsque -1 est un carré dans $L$.}

Rappelons le résultat suivant, prouvé dans $[\mathrm{DEK}]$ :

Théorème 4 : Soit $k$ un corps de caractéristique différente de 2. Soit $L / k$ une extension cyclique de degré $2^{r}$, avec $r \geq 3$, telle que $-1 \in L^{* 2}$. Soit $D$ un représentant de la classe de $\operatorname{disc}(L / k)$ modulo $k^{* 2}$.

Alors $\operatorname{Tr}_{L / k}(\langle 1\rangle) \simeq\langle D\rangle \perp\left(2^{r}-1\right)\langle 1\rangle$.

Corollaire 6 : Soit $k$ de caractéristique différente de 2 . Soit $L / k$ une extension cyclique de degré $n=2^{r} s$, avec $r \geq 3$ et $s \geq 1$ impair, telle que $-1 \in L^{* 2}$. Soit $\tau$ l'unique élément d'ordre 2 de son groupe de Galois, et soit $D$ un représentant de la classe de $\operatorname{disc}(L / k)$ modulo $k^{* 2}$. Alors $\operatorname{Tr}_{L / k}\left(\langle 1\rangle_{\tau}\right) \simeq s\langle 2,2 D\rangle \perp\left(2^{r-1} s-2 s\right)\langle 1\rangle \perp 2^{r-2} s \mathbb{H}$.

Preuve : Gardons les notations des remarques préliminaires (cf.§2). D'après [DEK], corollaire 4 , et une remarque précédente, on a

$\operatorname{Tr}_{K / k}(\langle 1\rangle) \simeq s\langle 2,2 D\rangle \perp\left(2^{r-1} s-2 s\right)\langle 1\rangle$. En utilisant la formule (2) et l'isomorphisme $\langle 2,2\rangle \simeq\langle 1,1\rangle$, on obtient par simplification de Witt que $\langle 2\rangle \otimes \operatorname{Tr}_{K / k}(\langle b\rangle) \simeq 2^{r-1} s\langle 1\rangle$. On conclue en utilisant $(3)$.

Corollaire 7 : Soit $k$ de caractéristique différente de 2 . Soit

$A=(a, L / k, \sigma)$ une algèbre cyclique de degré $n=2^{r} s$, avec $r \geq 3$ et $s \geq 1$ impair, telle que $-1 \in L^{* 2}$. Soit $D$ un représentant de la classe $\operatorname{de} \operatorname{disc}(L / k)$ modulo $k^{* 2}$. Alors

$\mathcal{T}_{A} \simeq s\langle 2,2 a, 2 D, 2 a D\rangle \perp\left(2^{r-1} s-2 s\right)\langle 1, a\rangle \perp \frac{n(n-1)}{2} \mathbb{H}$.

Preuve : On applique le corollaire 1 et le corollaire 6.

\section{Références}

[C] CONNER P.E., PERLIS R. A survey of trace forms of algebraic number fields. World Scientific Publ., Singapore (1984)

[D] DRAXL P.K. Skew fields. LMS Lecture Notes, No.83, Cambridge University Press (1983) 
[DEK] DREES C., EPKENHANS M., KRÜSKEMPER M. On the Computation of the Trace Form of Some Galois Extensions. Journal of Algebra 192, 209-234 (1997)

[EL] ELMAN R., LAM T.Y. Classification theorems for quadratic forms over fields. Comm.Math.Helv. 49, 373-381 (1974)

[J] JACOBSON A. Finite-Dimensional Division Algebras over Fields. Springer (1996)

[S] SCHARLAU W. Quadratic and Hermitian Forms. Grundlehren Math. Wiss. vol. 270. Springer-Verlag. New York (1985)

[Se] SERRE J.-P. Cohomologie galoisienne. Lecture notes in Mathematics 5, Springer-Verlag (1994)

[W] WATANABE Y. Trace forms of algebras. Math.Jap.34, No 4, 661-667 (1989) 